\title{
Evidence for Spatial Propagation of Photon-like Polaritons Generated by Exciton-Exciton Scattering in a GaAs/AlAs Multiple-Quantum-Well Structure
}

Yoshiaki Furukawa, Masaaki Nakayama

\begin{tabular}{|c|l|}
\hline Citation & Journal of the Physical Society of Japan, 87(9); 094718 \\
\hline Issue Date & $2018-09-03$ \\
\hline Type & Journal Article \\
\hline Textversion & Author \\
\hline Rights & $\begin{array}{l}\text { C2018 The Physical Society of Japan. The following article has been accepted by } \\
\text { Journal of the Physical Society of Japan. After it is published, it will be found at } \\
\text { https://doi.org/10.7566/JPSJ.87.094718. }\end{array}$ \\
\hline DOI & $10.7566 /$ JPSJ.87.094718 \\
\hline
\end{tabular}

Self-Archiving by Author(s)

Placed on: Osaka City University 


\title{
Evidence for Spatial Propagation of Photon-like Polaritons Generated by Exciton-Exciton Scattering in a GaAs/AlAs Multiple-Quantum-Well
}

\section{Structure}

\author{
Yoshiaki Furukawa and Masaaki Nakayama* \\ Department of Applied Physics and Electronics, Graduate School of Engineering, \\ Osaka City University, Sugimoto, Sumiyoshi-ku, Osaka 558-8585, Japan
}

We have investigated the polariton characteristics of the photoluminescence (PL) due to exciton-exciton scattering at $10 \mathrm{~K}$ in a GaAs/AlAs multiple-quantum-well structure with the use of spatially-resolved PL spectroscopy. We found that the PL band from the exciton-exciton scattering, the so-called P emission, is detected only at spots along a sample edge which is spatially separated from an excitation spot. In contrast, the PL bands attributed to the exciton and biexciton were observed only at the excitation spot. Because the exciton-exciton scattering occurs at the excitation spot, the appearance of the spatially-separated P-emission spot demonstrates that the photon-like lower polariton, which is the final state of the exciton-exciton scattering process, propagates along the interface, leading to conversion to a photon. The spectral shape of the P-PL band detected at the spatially-separated spot exhibits a fringe pattern, which is quantitatively explained by the Fabry-Pérot interference originating from the spatial separation of the P-emission and excitation spots. 


\section{Introduction}

Inelastic scattering processes of excitons with elementary excitations such as excitons, phonons, and free carriers are prominent phenomena leading to stimulated emission in an intermediate excitation-density regime below a Mott transition density. ${ }^{1)}$ As schematically shown in Fig. 1, in the inelastic scattering process of two excitons with a hydrogenic quantum number $n=1$ under the energy and momentum conservation, the so-called exciton-exciton scattering, one exciton is scattered into an $n \geq 2$ state including the continuum state with $n=\infty$, while the other is scattered onto the photon-like region in the lower polariton (LP) branch, and then the photon-like LP is converted to a photon, the so-called P emission. Thus, the photon-like LP is generated as the final state for the $\mathrm{P}$ emission. The $\mathrm{P}$ emission has been mainly observed in wide-gap semiconductors with large exciton binding energies such as $\mathrm{ZnO},{ }^{2-5)} \mathrm{CdS},{ }^{6}$ ) lightly-alloyed $\mathrm{Zn}_{x} \mathrm{Mg}_{1-x} \mathrm{O},{ }^{7)} \mathrm{GaN}^{8-11)}$ lightly-alloyed $\operatorname{In}_{x} \mathrm{Ga}_{1-x} \mathrm{~N},{ }^{12)}$ and $\mathrm{CuI} .{ }^{13-15)}$ The exciton-exciton scattering in quantum-well (QW) systems was reported in $\mathrm{Zn}_{x} \mathrm{Cd}_{1-x} \mathrm{Se} / \mathrm{ZnSe},{ }^{16,17)} \mathrm{ZnO} / \mathrm{Zn}_{x} \mathrm{Mg}_{1-x} \mathrm{O},{ }^{18)}$ and GaAs/AlAs ${ }^{19-21)}$ multiple-QW (MQW) structures. In the QW system, the exciton binding energy is enhanced by the quantum confinement effect, which leads to enhancement of the stability of the excitonic system. As a result, the P emission becomes observable in GaAs/AlAs MQW ${ }^{19-21)}$ in contrast to non-observable in a GaAs bulk crystal.

In the framework of the exciton-polariton picture, the exciton-exciton scattering process generates the photon-like LP as the final state as described above. The photon-like LP propagates in principle through a sample, and then is converted to a photon when it reaches the sample surface and/or edge at which the translational crystal symmetry is broken. ${ }^{22,23)}$ This is one of key characteristics of the exciton-exciton scattering; however, there have been limited works related to the polariton characteristics. ${ }^{420)}$ In these previous works, it was revealed that the energy dependence of the photoluminescence (PL) decay rate of the $\mathrm{P}$ emission is scaled by that of the group velocity of the photon-like LP; namely, the decay rate, $1 / \tau$, is proportional to the 
LP group velocity, $v_{\mathrm{g}}$, at a given energy: $1 / \tau(E)=A v_{\mathrm{g}}(E)$. In the polariton picture, the proportional constant should be $A=1 / L_{\mathrm{p}}$ if we neglect reflection at the sample surface and/or edge, where $L_{\mathrm{p}}$ is the polariton propagation length for the conversion to a photon. The value of $A$, however, largely deviates from $1 / L_{\mathrm{p}}$ in the previous works. ${ }^{4,20)}$ For example, in a $\mathrm{ZnO}$ thin film with a thickness of $4 \mu \mathrm{m},{ }^{4)}$ the value of $A$ was $35 \mathrm{~cm}^{-1}$ $\left(1 / A=2.9 \times 10^{2} \mu \mathrm{m}\right)$ in spite of that $L_{\mathrm{p}}$ is expected to be the film thickness. Thus, the proportional relation between $1 / \tau$ and $v_{\mathrm{g}}$ is phenomenological. It should be noted that the direct evidence for the polariton nature is to reveal the spatial propagation of the photon-like LP leading to the P emission. In a QW system, the spatial propagation of the photon-like LP is limited to the in-plane direction; therefore, it is expected that the conversion from the photon-like LP to the P emission occurs at a sample edge. This is advantageous in investigation of spatial propagation of the photon-like LP.

In the present work, we have focused on the spatially-resolved PL properties of exciton-exciton scattering at $10 \mathrm{~K}$ in a GaAs/AlAs MQW structure. Spatially-resolved PL spectroscopy provided the information for spatial propagation of the photon-like LP and conversion to the $\mathrm{P}$ emission. The key finding is the fact that the P-PL band is observed only at spots along a sample edge which is spatially separated from an excitation spot. In contrast, the exciton and biexciton PL bands are observed only at the excitation spot. This finding is the direct evidence for the polariton nature of the $\mathrm{P}$ emission. In other words, the photon-like LP generated by the exciton-exciton scattering at the excitation spot propagates along the interface, leading to the $\mathrm{P}$ emission at the spatially-separated spot. The spectral profile of the P-PL band detected at the spatially-separated spot exhibits a fringe pattern. The fringe pattern is quantitatively analyzed in the framework of the Fabry-Pérot interference due to the spatial separation between the P-emission and excitation spots, taking account of the energy dependence of the refractive index of the MQW sample.

\section{Experimental Details}


The sample used was a GaAs $(10 \mathrm{~nm}) / \mathrm{AlAs}(10 \mathrm{~nm}) \mathrm{MQW}$ structure with 30 periods grown on a (001) GaAs substrate at $560^{\circ} \mathrm{C}$ by molecular beam epitaxy. The polariton profile related to the $\mathrm{P}$ emission of this sample was already described in Ref. 20, where the energy dependence of the decay rate of the $\mathrm{P}$ emission was phenomenologically scaled by that of the group velocity of the photon-like LP calculated using an equation for the polariton dispersion relation. The above finding demonstrates that the P emission originates from the photon-like LP, which is closely related to the main issue in the present work.

The schematic diagram of the measurement system for spatially-resolved PL spectroscopy is shown in Fig. 2(a). The excitation light source in the PL measurement was a mode-locked Ti:sapphire pulse laser with a pulse duration of $110 \mathrm{fs}$ and a repetition rate of $76 \mathrm{MHz}$. The peak energy of the excitation laser was fixed at $1.717 \mathrm{eV}$ which is far above the fundamental exciton energies. We used a refractive beam shaper in combination with a beam expander to produce a flat-top shape of the excitation laser beam according to Ref. 24, which considerably reduced spatial inhomogeneity of a photogenerated exciton density. An objective lens with a numerical aperture of 0.40 and a focal length of $10 \mathrm{~mm}$ was used for the excitation and collection of PL. The PL from the sample was focused on a complementary metal-oxide semiconductor (CMOS) profiler to observe a spatial PL image. In this case, a pinhole was removed from the light path. The magnification of the PL image at the focal plane was 20 times, which was calibrated using a micro-ruler. Figure 2(b) shows the spatial PL-intensity profile observed with the CMOS profiler under a weak excitation condition. It is obvious that the spatial profile exhibits a flat-top shape. We defined a diameter of $52 \mu \mathrm{m}$, within which $86.5 \%$ corresponding to $1-1 / e^{2}$ of the total intensity is covered, as a measure of the excitation-beam spot size. In the measurement of spatially-resolved PL spectra, the PL image was spatially selected using a pinhole with a diameter of $50 \mu \mathrm{m}$ at the focal plane in the light path as shown in Fig. 2(a): The spatial resolution was $2.5 \mu \mathrm{m}$. The position of the pinhole was monitored using the CMOS profiler. A cooled charge 
coupled device attached to a 32-cm single monochromator with a spectral resolution of $0.10 \mathrm{~nm}$ was used to measure PL spectra. In addition, we observed spatially-integrated PL spectra. The experimental setup was the same as that for spatially-resolved PL spectroscopy shown in Fig. 2(a); however, the pinhole at the focal plane was removed. Furthermore, a PL excitation (PLE) spectrum was measured to characterize the energies of the first quantized heavy-hole (H11) and light-hole (L11) excitons. The excitation light was produced by combination of a tungsten lamp and a 32-cm single monochromator with a spectral resolution of $0.2 \mathrm{~nm}$. The PL was dispersed by a $25-\mathrm{cm}$ double monochromator with a spectral resolution of $0.3 \mathrm{~nm}$ and detected using a photon counting system. All the optical measurements were performed at $10 \mathrm{~K}$ with the use of a low-vibration-type closed-cycle helium cryostat: The vibration amplitude was $\sim 2 \mu \mathrm{m}$.

\section{Results and Discussion}

To confirm the overall PL properties, Fig. 3 shows the spatially-integrated PL spectra at various excitation fluences at $10 \mathrm{~K}$ in the GaAs $(10 \mathrm{~nm}) / \mathrm{AlAs}(10 \mathrm{~nm}) \mathrm{MQW}$, where the maximum excitation fluence was $F_{0}=50 \mu \mathrm{J} / \mathrm{cm}^{2}$ and the intensity of each spectrum was normalized to the maximum value. The black dashed curve on the top indicates the PLE spectrum detected at $1.564 \mathrm{eV}$ to evaluate the H11- and L11-exciton energies. The PLE spectrum on the low energy side of the H11-exciton band could not be measured because of Rayleigh scattering by the excitation light. The blue dotted line labeled $E_{\mathrm{H} 11}$ indicates the H11-exciton energy. The exciton-PL band labeled X is mainly observed at the lowest excitation fluence of $3 \times 10^{-5} F_{0}$. The slight difference between the energies of the X-PL peak and H11 exciton results from weak localization of the exciton due to interface fluctuations. With an increase in excitation fluence, the PL band labeled M continuously grows on the low energy side of the X-PL band. The low energy tail of the M-PL band is a typical feature of the biexciton PL, the so-called inverse-Boltzmann line shape. The biexciton binding energy in a 10-nm thick GaAs QW was reported to be $2.0 \mathrm{meV}$ using degenerate four wave mixing spectroscopy. ${ }^{25)}$ The energy spacing 
between the $\mathrm{X}$ and M PL bands almost corresponds to the biexciton binding energy.

At an excitation fluence of $0.2 F_{0}\left(\sim 10 \mu \mathrm{J} / \mathrm{cm}^{2}\right)$, a new PL band labeled P appears with a threshold-like nature, and then its intensity relative to the X-and M-PL bands markedly increases with an increase in excitation fluence. The threshold-like appearance of the P-PL band suggests the occurrence of optical gain producing the stimulated emission, which is one of the typical characteristics of the exciton-exciton scattering. ${ }^{1)}$ The occurrence of optical gain was experimentally confirmed in various semiconductors. ${ }^{8,12,13,17,19)}$ The mechanism of stimulated emission is theoretically explained in Ref. 26 in the framework of the second quantization for creation and annihilation of excitons and photons in the exciton-exciton scattering process. Taking account of the momentum and energy conservation in the exciton-exciton scattering process, the peak energy of the P-PL band is given by ${ }^{2)}$

$$
E_{\mathrm{p}}=E_{\mathrm{X}, n=1}-\left(E_{\mathrm{X}, n \geq 2}-E_{\mathrm{X}, n=1}\right)-3 \sigma k_{\mathrm{B}} T_{e f f},
$$

where $E_{\mathrm{X}, n}$ is the exciton energy with a hydrogenic quantum number $n, T_{\text {eff }}$ is an effective temperature, and $\sigma$ is a positive constant smaller than unity. The last term in Eq. (1) corresponds to an excess kinetic energy. The red dotted line labeled $E_{\mathrm{p}}$ in Fig. 3 indicates the calculated P-PL energy on the assumption of the scattering process into the $n=2$ exciton state, the so-called $\mathrm{P}_{2}$ emission, where the energy spacing between the $n=1$ and $n=2 \mathrm{H} 11$ excitons was taken from Ref. 27: $E_{\mathrm{X}, n=2}-E_{\mathrm{X}, n=1}=8.9 \mathrm{meV}$. Here, the term of the effective temperature, $-3 \sigma k_{\mathrm{B}} T_{\text {eff, }}$, in Eq. (1) is negligible at the threshold excitation fluence at $10 \mathrm{~K}$, which was confirmed from the following fact observed in the P-PL dynamics. $^{20)}$ The temporal energy of the P-PL band reflecting a change of $T_{\text {eff }}$ in the decay process was almost constant and consistent with the $\mathrm{P}_{2}$-emission energy without $-3 \sigma k_{\mathrm{B}} T_{\text {eff. }}$ The calculated energy $E_{\mathrm{p}}$ is in agreement with the peak energy of the P-PL band at the threshold in Fig. 3. The P-PL band exhibits a low energy shift with an increase in excitation fluence from the threshold, which reflects an increase in effective temperature. 
Figure 4 shows the spatial PL images at the excitation fluences of (a) 0.10 $\mu \mathrm{J} / \mathrm{cm}^{2}$ and (b) $40 \mu \mathrm{J} / \mathrm{cm}^{2}$ at $10 \mathrm{~K}$, where the PL intensity is shown by the color scale. The black dashed line indicates the sample edge and the white-colored area corresponds to the outside of the sample. At $0.10 \mu \mathrm{J} / \mathrm{cm}^{2}$, which is much lower than the threshold excitation fluence for the P-PL band shown in Fig. 3, only the PL spot corresponding to the excitation spot is observed. In contrast, at the excitation fluence above the threshold, $40 \mu \mathrm{J} / \mathrm{cm}^{2}$, we notice appearance of new PL spots along the sample edge spatially separated from the excitation spot. This fact is a key issue in this work.

To reveal the origin of the spatially-separated PL spot, we measured the excitation fluence dependence of spatially-resolved PL spectra. Figure 5 shows the spatially-resolved PL spectra detected at (a) the center of the excitation spot and (b) the spatially-separated spot at various excitation fluences, where the maximum excitation fluence was $F_{0}=50 \mu \mathrm{J} / \mathrm{cm}^{2}$ and the intensity of each spectrum was normalized to the maximum value. We selected the strongest PL area at the spatially-separated spot along the sample edge. The blue and red dotted lines indicate the H11-exciton and $\mathrm{P}_{2}$-emission energies, respectively. It is evident that the PL spectral profile at the spatially-separated spot is fully different from that at the excitation spot. The striking fact is that the P-PL band does not exist at the excitation spot. Here, we mention the measurement of spatially-integrated PL spectra shown in Fig. 3. It is evident from Fig. 4(b) that the total PL intensity at the excitation spot is much stronger than that at the spatially-separated spot. In the measurement of spatially-integrated PL spectra, we adjusted the optical configuration for PL detection to highlight the appearance of the P-PL band.

It is obvious from Fig. 5(b) that only the P-PL band is observed at the spatially-separated spot. The disappearance of the X- and M-PL bands can be attributed to the reabsorption effect on the PL intensities through the distance between the excitation and spatially-separated spots. Note that the energy region of the P-PL band overlaps with that of the M-PL band; however, only the M-PL band disappears at the spatially-separated spot. This fact indicates that the reabsorption effect on the P-PL band 
is negligible. As a matter of course, the exciton-exciton scattering occurs at the excitation spot. If the polariton nature, which corresponds to spatial propagation of the photon-like LP, would be negligible, the P-PL band should appear at the excitation spot. From Fig. 5(a), however, it is evident that the P-PL band could not be observed at the excitation spot in spite of that the X-and M-PL bands are observable. This fact indicates that the conversion from the photon-like LP to the $\mathrm{P}$ emission never occurs at the excitation spot. In other words, the spatial propagation of the photon-like LP arriving at the sample edge is essential for appearance of the $\mathrm{P}$ emission. Therefore, the above results for the spatially-resolved PL spectra can be explained by the following three processes: (1) generation of the photon-like LP by the exciton-exciton scattering at the excitation spot, (2) propagation of the photon-like LP from the excitation spot along the interface, and (3) conversion from the photon-like LP to the P emission at the sample edge. The conversion from the photon-like LP to the $\mathrm{P}$ emission is caused by the breakdown of the translational crystal symmetry at the sample edge. In the spatial PL image shown in Fig. 4(b), the P-PL intensity at the spatially-separated spot exhibits the position dependence. A possible reason for the position dependence is as follows. Defects also contribute to the conversion from the photon-like LP to a phonon. The density of defects usually has spatial distribution. Thus, a higher defect density leads to a stronger P-PL intensity. In addition, the influence of micro-cracks, which tend to exist at the sample edge because of cleavage of sample piece, on the photon conversion is similar to that of defects. We confirmed that the P-PL intensity at the spatially-separated spot is sensitively changed by moving the excitation spot parallel to the sample edge. Figure 4(b) corresponds to the most prominent PL image for the spatially-separated spot. Note that the spatially-resolved P-PL band is observed in the excitation fluence region lower than the threshold in the spatially-integrated PL spectra shown in Fig. 3. As described above, the threshold-like appearance of the P-PL band results from occurrence of the stimulated $\mathrm{P}$ emission. The spontaneous $\mathrm{P}$ emission is also observable in principle. ${ }^{26)}$ Because the PL intensity of the spontaneous P emission is considerably 
weak, the low energy tail of the M-PL band prevents the observation of the P-PL band below the threshold in the spatially-integrated PL spectra.

The P-PL band detected at the spatially-separated spot exhibits a fringe pattern as shown in Fig. 5(b). Such a fringe pattern usually originates from interference due to multiple reflections between two interfaces, the so-called Fabry-Pérot interference. The quantitative analysis of the fringe pattern, which is described below, is significant in clarifying how the appearance of the spatially-separated spot affects the P-PL spectral shape. The wavelength separation between two adjacent peaks, $\Delta \lambda$, is given by ${ }^{28)}$

$$
\Delta \lambda=\frac{\lambda /(2 L)}{n / \lambda-d n / d \lambda},
$$

where $\lambda$ is a wavelength, $L$ is a distance between two interfaces, and $n$ is a refractive index. Because the P-PL band appears close to the H11 exciton, the wavelength dispersion of $n$ due to the excitonic transition should be considered. To discuss quantitatively the fringe pattern, we calculated the energy dependence of the refractive index around the H11-exciton energy. According to Ref. 29 in the framework of an effective dimension approximation, the complex dielectric function around the exciton energy in a $\mathrm{QW}, \varepsilon_{\mathrm{QW}}(E)$, is given by

$$
\begin{aligned}
& \varepsilon_{\mathrm{QW}}(E)=\frac{S \times R^{d / 2-1}}{(E+i \gamma)^{2}}[g(\xi(E+i \gamma))+g(\xi(-E-i \gamma))-2 g(\xi(0))], \\
& g(\xi)=\frac{2 \pi \Gamma(D / 2+\xi)}{\Gamma(D / 2)^{2} \Gamma(1-D / 2+\xi) \xi^{d-2}}[\cot \pi(D / 2-\xi)-\cot \pi D], \\
& \xi(z)=\sqrt{R /\left(E_{\mathrm{g}}-z\right)}
\end{aligned}
$$

where $d$ is the effective dimension, ${ }^{30)} S$ is a constant related to the exciton oscillator strength, $R$ is the exciton binding energy in a bulk crystal, $E_{\mathrm{g}}$ is the band-gap energy of the QW, $\gamma$ is the broadening factor, $D=d-1$, and $\Gamma(x)$ is the gamma function. The energy dependence of the background dielectric constant was approximately treated using the Sellmeier equation. ${ }^{31)}$ The total dielectric function for a QW including the background dielectric constant is described as 


$$
\varepsilon_{\text {total }}(E)=A+\frac{C}{1-B E^{2}}+\varepsilon_{\mathrm{QW}}(E)
$$

where $A, B$, and $C$ are the constants for the Sellmeier equation: $A=7.10, B=0.18 \mathrm{eV}^{-2}$, and $C=3.78$ in GaAs. ${ }^{31)}$ The value of $d$ was taken from Ref. $30: d=2.4$ for a 10-nm thick GaAs QW. The value of $R$ is $4.2 \mathrm{meV}$ in GaAs. ${ }^{32)}$ According to Ref. 33, the value of $S$ is $1.0 \mathrm{eV}^{1.8}$ in $d=2.4$. The values of $E_{\mathrm{g}}$ and $\gamma$ were estimated from the line-shape analysis of the PLE spectrum with the use of an absorption spectrum calculated using $2 k_{0} \times \operatorname{Im}\left[\varepsilon_{\mathrm{QW}}(E)^{1 / 2}\right]$, where $k_{0}$ is the wave vector of light in vacuum. We assumed that the shape of the PLE spectrum in the vicinity of the H11 exciton is consistent with that of the absorption spectrum. Figure 6(a) shows the result of the line-shape analysis of the H11-exciton band in the PLE spectrum on the basis of the theory described above, where the black solid circle and red solid curve indicate the experimental and fitted spectra, respectively. The fitted result reproduces the experimental PLE spectrum for the H11 exciton, which indicates the validity of the theory used in this work. The values of the fitting parameters are as follows: $E_{\mathrm{g}}=1.5770 \mathrm{eV}$ and $\gamma=2.4 \mathrm{meV}$.

Figures 6(b) and 6(c) show the calculated values of $n$ and $d n / d \lambda$, respectively, as a function of energy. In the long wavelength approximation, the value of $n$ in the MQW is given by the layer-thickness weighted average of the refractive indices of the GaAs QW, $n_{\mathrm{W}}=\operatorname{Re}\left[\varepsilon_{\text {total }}(E)^{1 / 2}\right]$, and the AlAs barrier, $n_{\mathrm{B}}: n=\left(n_{\mathrm{W}} L_{\mathrm{W}}+n_{\mathrm{B}} L_{\mathrm{B}}\right) /\left(L_{\mathrm{W}}+L_{\mathrm{B}}\right)$, where $L_{\mathrm{W}}$ and $L_{\mathrm{B}}$ are the thicknesses of the GaAs and AlAs layers, respectively. For the present MQW sample, the refractive index is given by $n=\left(n_{\mathrm{w}}+n_{\mathrm{b}}\right) / 2$ because of $L_{\mathrm{W}}=L_{\mathrm{B}}$. The value of $n_{\mathrm{B}}$ was treated as a constant because the energy region of the P-PL band is far below the band-gap energy of AlAs: $n_{\mathrm{B}}=3.007$ at $1.559 \mathrm{eV}{ }^{34)}$ Figure $6(\mathrm{~d})$ shows the PL spectrum detected at the spatially-separated spot at the excitation fluence of $0.05 F_{0}$, where $F_{0}$ is the same as that in Fig. 5(b). In Fig. 5(b), at the excitation fluences of $0.05 F_{0}, 0.1 F_{0}$, and $0.3 F_{0}$, at which the energy and width of the P-PL band are almost constant, the visibility of the fringe pattern is almost unchanged. At $0.5 F_{0}$ and $F_{0}$, the P-PL band exhibits a low energy shift and broadening, which may modify the visibility 
of the fringe pattern mainly because of the energy dependence of the refractive index. The inset of Fig. 6(d) shows the magnified P-PL band in the wavelength region around the peak, where the wavelength spacing of the fringe pattern is $\Delta \lambda=0.59 \mathrm{~nm}$. At the peak energy of the P-PL band, $1.5594 \mathrm{eV}, n$ and $d n / d \lambda$ were obtained to be 3.40 and $-8.0 \times 10^{-3}$ $\mathrm{nm}^{-1}$ from Figs. 6(b) and 6(c), respectively. Consequently, $L$ was estimated to be $55 \mu \mathrm{m}$ using Eq. (2). In Fig. 4(b), the position of the spatially-separated spot with the strongest PL intensity is $\sim 63 \mu \mathrm{m}$ away from the right-side edge of the excitation spot. Here, we define the edge of the excitation spot as the boundary of the red-colored area in Fig. 4(b). The calculated and experimental values of $L$ are almost in agreement with each other in spite of the troublesome calculation of the refractive index. Therefore, the fringe pattern of the P-PL band is attributed to the Fabry-Pérot interference between the sample edge and the right-side edge of the excitation spot. Note that the refractive index in the excitation spot is slightly smaller than that in the surrounding area because photogenerated excitons with a relatively high density reduce the exciton oscillator strength. ${ }^{35)}$ Thus, the refractive index difference between the inside and outside of the excitation spot results in formation of the interface for the Fabry-Pérot interference. The quantitative estimation of a change of the refractive index at the excitation spot is beyond the scope of this paper. The left side edge of the excitation spot can also contribute to the Fabry-Pérot interference. In this case, $L$ corresponds to $\sim 24 \mu \mathrm{m}$; therefore, $\Delta \lambda$ is estimated to be $\sim 1.4 \mathrm{~nm}$ using Eq. (2). Because $\Delta \lambda$ of $\sim 1.4 \mathrm{~nm}$ is about two-thirds of the full width at half maximum of the spatially-resolved P-PL band, the P-PL band itself may hide the relatively long fringe pattern originating from the left side edge of the excitation spot.

\section{Conclusions}

We have investigated the spatially-resolved PL properties from the viewpoint of the polariton characteristics of the $\mathrm{P}$ emission due to exciton-exciton scattering at 10 $\mathrm{K}$ in the GaAs $(10 \mathrm{~nm}) / \mathrm{AlAs}(10 \mathrm{~nm}) \mathrm{MQW}$ structure. From the spatially-resolved PL 
spectra at various excitation fluences, we found that only the P-PL band appears at the spatially-separated spot along the sample edge away from the excitation spot, while it is not observable at the excitation spot in spite of that the exciton and biexciton PL bands are observed. This fact demonstrates that the photon-like LP generated by the exciton-exciton scattering at the excitation spot propagates along the interface, and then is converted to a photon corresponding to the $\mathrm{P}$ emission at the sample edge. The P-PL band observed at the spatially-separated spot exhibits a fringe pattern. We analyzed the fringe pattern in the framework of the Fabry-Pérot interference, taking account of the refractive index dispersion due to the H11-exciton transition. As a result, we showed that the spatial separation of the P-emission and excitation spots causes the Fabry-Pérot interference.

Acknowledgement This work was supported by JSPS KEKENHI Grant Number $18 \mathrm{~K} 03494$. 
References

*nakayama@a-phys.eng.osaka-cu.ac.jp

1) For a review, C. Klingshirn and H. Haug, Phys. Rep. 70, 315 (1981).

2) C. Klingshirn, Phys. Status Solidi B 71, 547 (1975).

3) Z. K. Tang, G. K. L. Wong, P. Yu, M. Kawasaki, A. Ohtomo, H. Koinuma, and Y. Segawa, Appl. Phys. Lett. 72, 3270 (1998).

4) S. Wakaiki, H. Ichida, K. Mizoguchi, D. Kim, Y. Kanematsu, and M. Nakayama, Phys. Status Solidi C 8, 116 (2011).

5) S. Wakaiki, H. Ichida, T. Kawase, K. Mizoguchi, D. Kim, M. Nakayama, and Y. Kanematsu, Eur. Phys. J. B 86, 387 (2013).

6) K. Bohnert, G. Schmieder, and C. Klingshirn, Phys. Status Solidi B 98, 175 (1980).

7) C. H. Chia, J. N. Chen, and Y. M. Hu, Appl. Phys. Lett. 99, 131908 (2011).

8) J. Holst, L. Eckey, A. Hoffmann, I. Broser, B. Schöttker, D. J. As, D. Schikora, and K. Lischka, Appl. Phys. Lett. 72, 1439 (1998).

9) S. Bidnyk, T. J. Schmidt, B. D. Little, and J. J. Song, Appl. Phys. Lett. 74, 1 (1999).

10) S. Kurai, A. Kawabe, T. Sugita, S. Kubo, Y. Yamada, T. Taguchi, and S. Sakai, Jpn. J. Appl. Phys. 38, L102 (1999).

11) H. Tanaka, M. Ando, T. Uemura, and M. Nakayama, Phys. Status Solidi C 3, 3512 (2006).

12) M. Nakayama, R. Kitano, M. Ando, and T. Uemura, Appl. Phys. Lett. 87, 092106 (2005).

13) I. Tanaka and M. Nakayama, J. Appl. Phys. 92, 3511 (2002).

14) H. Ichida, Y. Kanematsu, T. Shimomura, K. Mizoguchi, D. Kim, and M. Nakayama, Phys. Rev. B 72, 045210 (2005).

15) H. Ichida, Y. Kanematsu, K. Mizoguchi, D. Kim, and M. Nakayama, Phys. Rev. B 76, 085417 (2007).

16) Y. Kawakami, I. Hauksson, H. Stewart, J. Simpson, I. Galbraith, K. A. Prior, and B. C. Cavenett, Phys. Rev. B 48, 11994 (1993). 
17) J. Y. Jen, T. Tsutsumi, I. Souma, Y. Oka, and H. Fujiyasu, Jpn. J. Appl. Phys. 32, L1542 (1993).

18) H. D. Sun, T. Makino, N. T. Tuan, Y. Segawa, Z. K. Tang, G. K. L. Wong, M. Kawasaki, A. Ohtomo, K. Tamura, and H. Koinuma, Appl. Phys. Lett. 77, 4250 (2000).

19) M. Nakayama, T. Hirao, and T. Hasegawa, J. Appl. Phys. 105, 123525 (2009).

20) Y. Furukawa, H. Takeuchi, and M. Nakayama, J. Phys. Soc. Jpn. 83, 054709 (2014).

21) S. Nakanishi, Y. Furukawa, and M. Nakayama, Phys. Status Solidi C 13, 109 (2016).

22) C. F. Klingshirn, Semiconductor Optics (Springer, Berlin, 2012) 4th ed., p. 507.

23) M. Bamba, S. Wakaiki, H. Ichida, K. Mizoguchi, D. Kim, M. Nakayama, and Y. Kanematsu, Phys. Rev. B 91, 235205 (2015).

24) A. Laskin and V. Laskin, Proc. SPIE 8490, 84900J (2012).

25) D. Birkedal, J. Singh, V. G. Lyssenko, J. Erland, and J. M. Hvam, Phys. Rev. Lett. 76, 672 (1996).

26) S. W. Koch, H. Haug, G. Schmieder, W. Bohnert, and C. Klingshirn, Phys. Status Solidi B 89, 431 (1978).

27) L. C. Andreani and A. Pasquarello, Phys. Rev. B 42, 8928 (1990).

28) D. C. Reynolds and T. C. Collins, Excitons: Their Properties and Uses, (Academic Press, New York, 1981) p. 120. Equation (5.23) in this book corresponds to Eq. (2).

29) C. Tanguy, P. Lefebvre, H. Mathieu, and R. J. Elliott, J. Appl. Phys. 82, 798 (1997).

30) H. Mathieu, P. Lefebvre, and P. Christol, Phys. Rev. B 46, 4092 (1992).

31) J. S. Blakemore, J. Appl. Phys. 62, 4528 (1987).

32) D. D. Sell, Phys. Rev. B 6, 3750 (1972).

33) M. V. Marquezini, J. Tignon, T. Hasche, and D. S. Chemla, Appl. Phys. Lett. 73, $2313(1998)$.

34) R. E. Fern and A. Onton, J. Appl. Phys. 42, 3499 (1971). The refractive index at $1.559 \mathrm{eV}$ was estimated from linear interpolation between 3.005 at $1.550 \mathrm{eV}$ and 3.009 at $1.573 \mathrm{eV}$ listed in Table I of this reference. 
35) K.-H. Schlaad, Ch. Weber, J. Cunningham, C. V. Hoof, G. Borghs, G. Weimann, W. Schlapp, H. Nickel, and C. Klingshirn, Phys. Rev. B 43, 4268 (1991). 
Figure captions

Fig. 1. (Color online) Schematic diagram of the exciton-exciton scattering process, where $E_{\mathrm{X}}$ is the $n=1$ transverse exciton energy at the $\Gamma$ point and $E_{\mathrm{g}}$ is the band-gap energy.

Fig. 2. (Color online) (a) Schematic diagram of the measurement system for spatially-resolved PL spectroscopy and (b) spatial PL-intensity profile observed with a CMOS profiler under a weak excitation condition.

Fig. 3. (Color online) Spatially-integrated PL spectra at various excitation fluences at 10 $\mathrm{K}$ in the GaAs $(10 \mathrm{~nm}) / \mathrm{AlAs}(10 \mathrm{~nm}) \mathrm{MQW}$, where the maximum fluence is $F_{0}=50$ $\mu \mathrm{J} / \mathrm{cm}^{2}$ and the intensity of each PL spectrum is normalized to the maximum one. The black dashed curve on the top depicts the PLE spectrum for the reference of the H11and L11-exciton energies. The vertical dotted lines labeled $E_{\mathrm{H} 11}$ and $E_{\mathrm{P}}$ indicate the H11-exciton and $\mathrm{P}_{2}$-emission energies, respectively.

Fig. 4. (Color online) Spatial PL images at the excitation fluences of (a) $0.10 \mu \mathrm{J} / \mathrm{cm}^{2}$ and (b) $40 \mu \mathrm{J} / \mathrm{cm}^{2}$, where the PL intensity is indicated by the color scale. The black dashed line indicates the sample edge.

Fig. 5. (Color online) Spatially-resolved PL spectra at various excitation fluences detected at (a) the center of the excitation spot and (b) the spatially-separated spot, where the maximum excitation fluence is $F_{0}=50 \mu \mathrm{J} / \mathrm{cm}^{2}$ and the intensity of each PL spectrum is normalized to the maximum one. The vertical dotted lines labeled $E_{\mathrm{H} 11}$ and $E_{\mathrm{P}}$ indicate the $\mathrm{H} 11$-exciton and $\mathrm{P}_{2}$-emission energies, respectively.

Fig. 6. (Color online) (a) Result of the line-shape analysis of the H11-exciton band in 
the PLE spectrum based on the theoretical model for the dielectric function given by Eq. (3), where the black solid circle and red solid curve indicate the experimental and fitted spectra, respectively. (b) Calculated refractive index $n$ and (c) $d n / d \lambda$ as a function of energy using Eqs. (3) and (6). (d) PL spectrum detected at the spatially-separated spot at the excitation fluence of $0.05 F_{0}$, where $F_{0}$ is the same as that in Fig. 5(b). The inset shows the magnified PL spectrum in the wavelength region around the peak, where $\Delta \lambda$ indicates the wavelength spacing of the fringe pattern. 


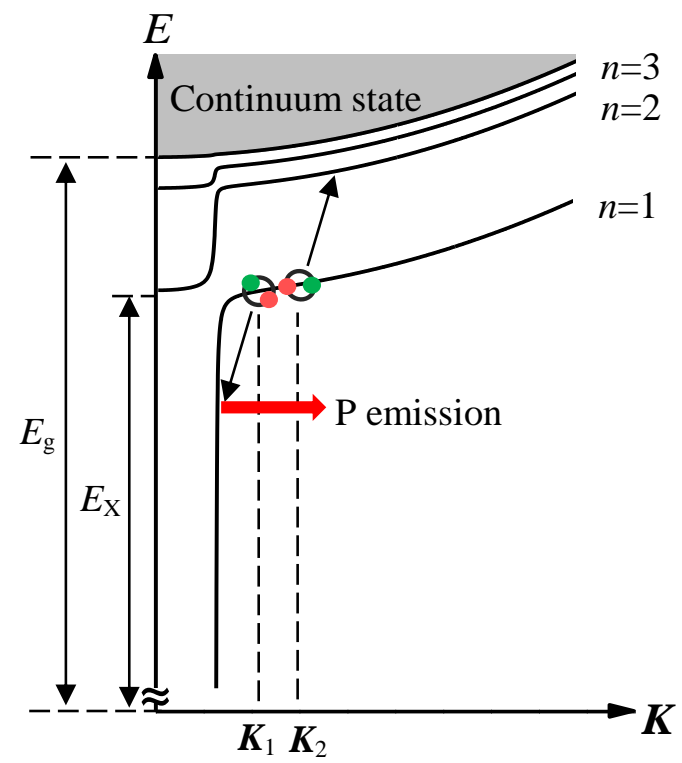

Fig. 1 

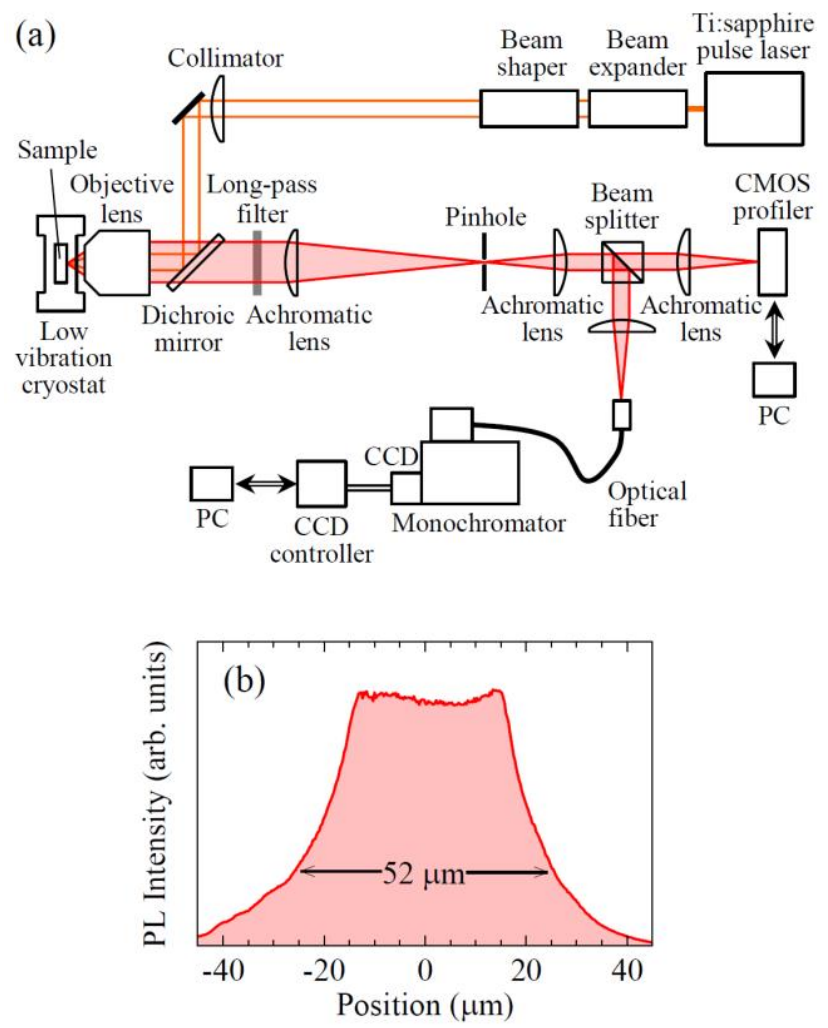

Fig. 2 


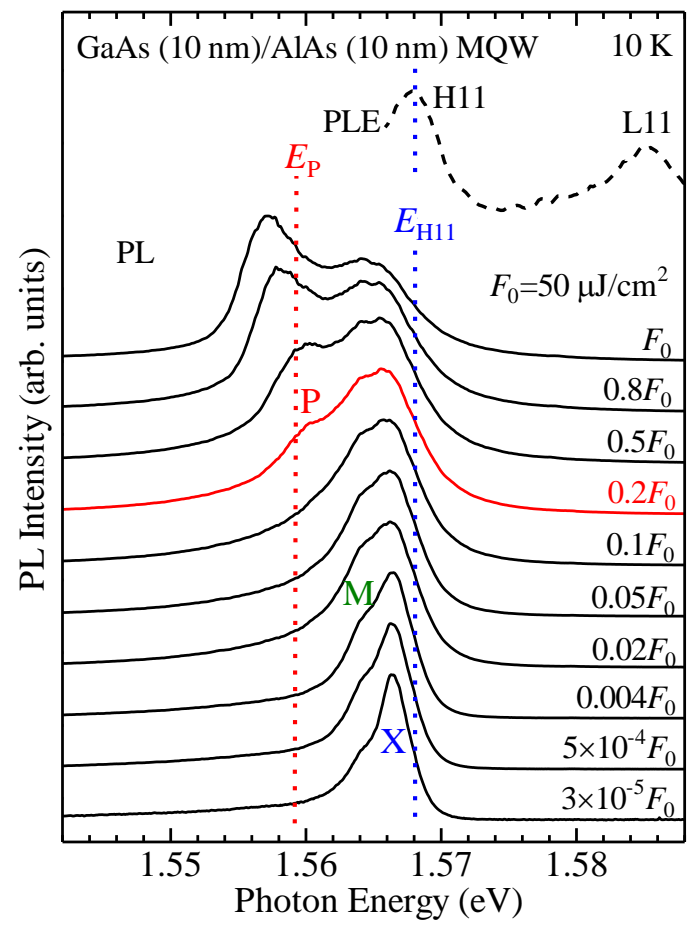

Fig. 3 

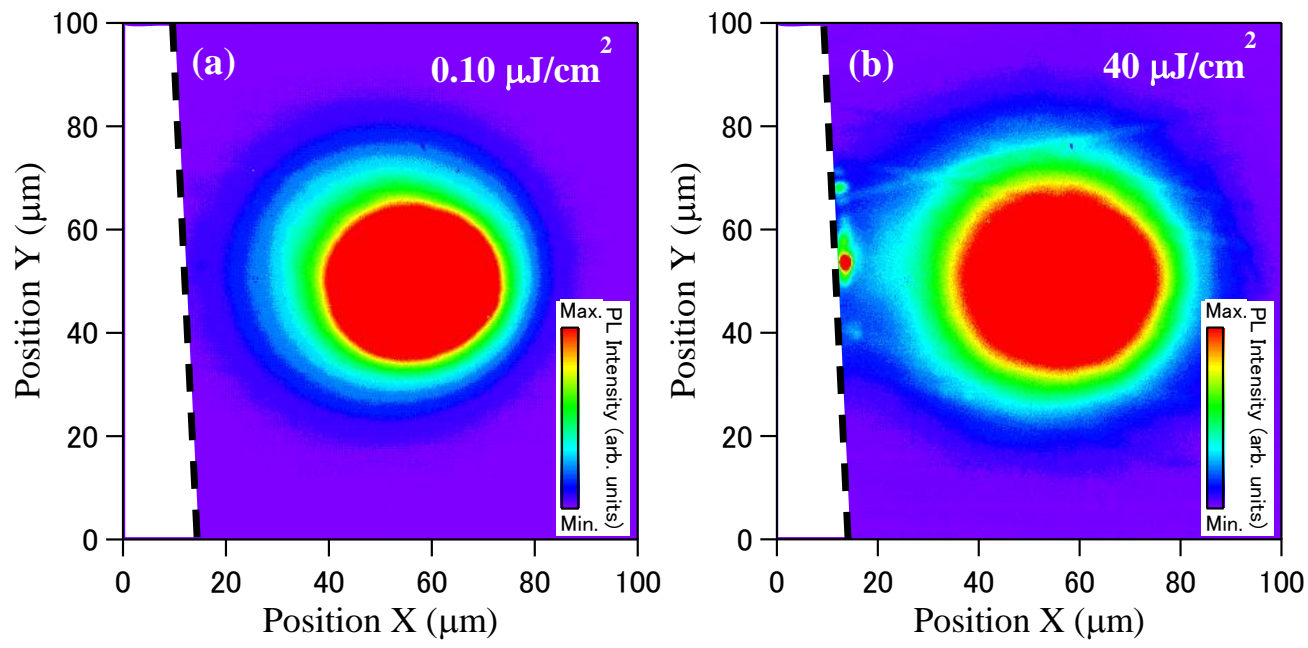

Fig. 4 


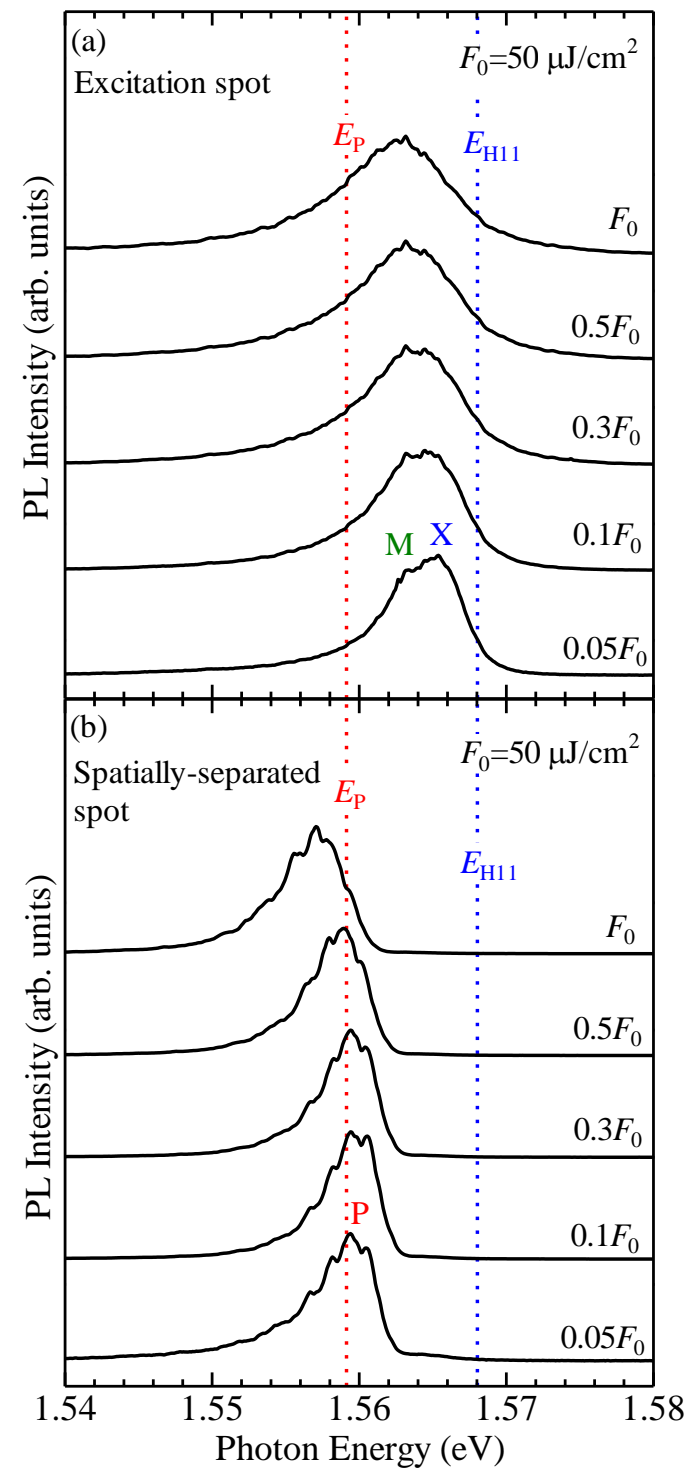

Fig. 5 


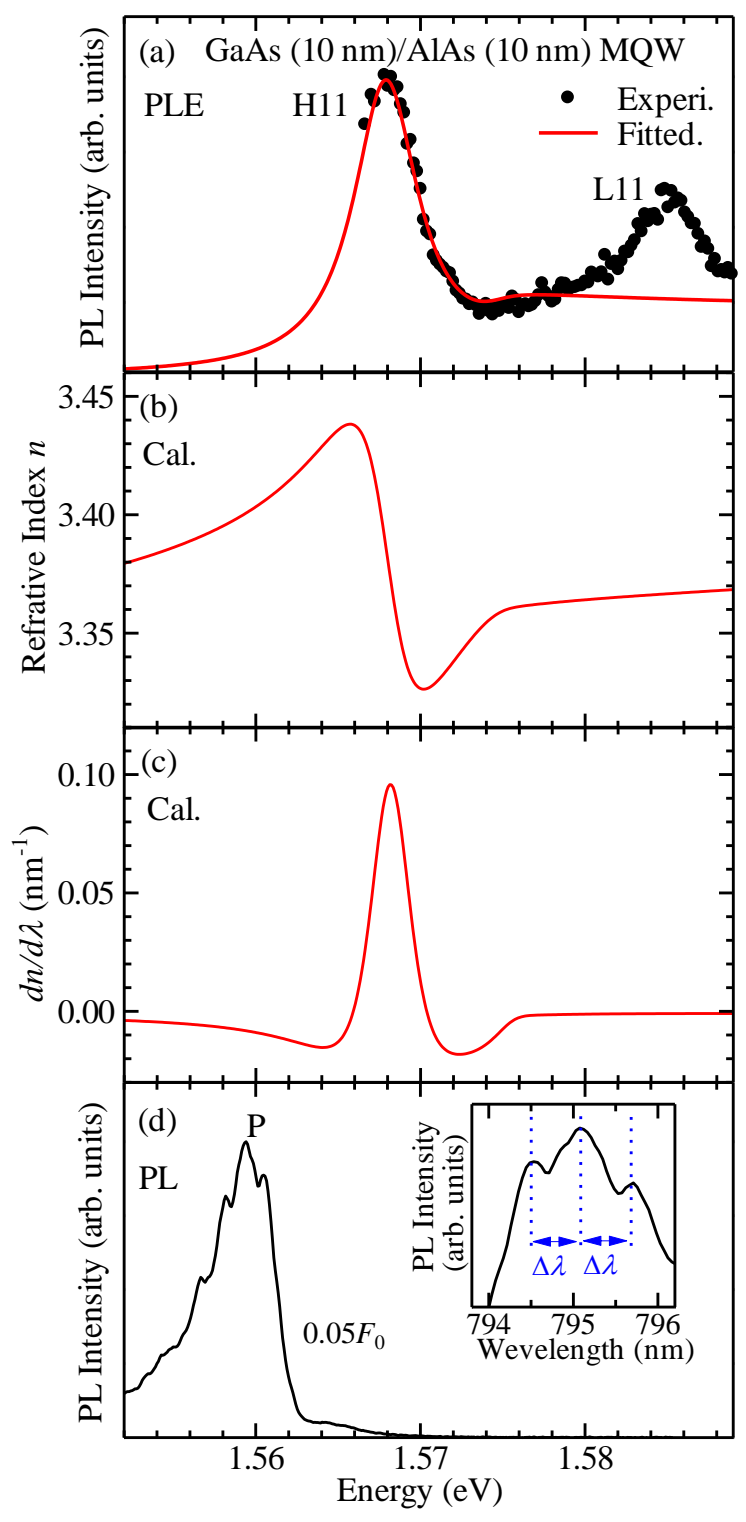

Fig. 6 